\title{
The CMS electromagnetic calorimeter barrel upgrade for High-Luminosity LHC
}

\author{
Michael Planer*i \\ University of Notre Dame \\ 225 Nieuwland Science Hall, Notre Dame, IN 46556, USA \\ E-mail: mplaner@cern.ch
}

The High Luminosity LHC (HL-LHC) will provide unprecedented instantaneous and integrated luminosity. The lead tungstate crystals forming the barrel part of the CMS Electromagnetic Calorimeter (ECAL) will still perform well, even after the expected $3000 \mathrm{fb}^{-1}$ at the end of HLLHC. The avalanche photodiodes (APDs) used to detect the scintillation light have recently been exposed to the levels of radiation expected at the end of HL-LHC. Although they will continue to be operational, there will be some increase in noise due to radiation-induced dark-currents. Triggering on electromagnetic objects with 140 pileup events necessitates a change of the front-end electronics. New developments in high-speed optical links will allow single-crystal readout at 40 $\mathrm{MHz}$ to upgraded off-detector processors, allowing maximum flexibility and enhanced triggering capabilities. The very-front-end system will also be upgraded, to provide improved rejection of anomalous signals in the APDs as well as to mitigate the increase in APD noise. We are also considering lowering the ECAL barrel operating temperature from 18 degrees $\mathrm{C}$ to about 8-10 degrees $\mathrm{C}$, in order to increase the scintillation light output and reduce the APD dark current.

Technology and Instrumentation in Particle Physics 2014,

2-6 June, 2014

Amsterdam, the Netherlands

\footnotetext{
${ }^{*}$ Speaker.

$\dagger$ on behalf of the CMS Collaboration
} 


\section{Introduction}

The Compact Muon Solenoid (CMS) [1] detector was originally designed to operate for about ten years, for LHC instantaneous/integrated luminosities of $1 \times 10^{34} \mathrm{~cm}^{-2} \mathrm{~s}^{-1}$ and $500 \mathrm{fb}^{-1}$ respectively. The High Luminosity LHC (HL-LHC) will provide an instantaneous luminosity of $5 \times 10^{34}$ $\mathrm{cm}^{-2} \mathrm{~s}^{-1}$; CMS will accumulate an integrated luminosity of $3000 \mathrm{fb}^{-1}$ by about 2035 [2]. Requirements on the triggering system of CMS will necessitate changes in the on-detector electronics of the ECAL. Upgrading these electronics also allows for upgrades to improve performance in this challenging environment.

\subsection{CMS electromagnetic calorimeter}

The CMS electromagnetic calorimeter (ECAL) is a hermetic, homogenous, high granularity and high resolution detector composed of PbWO4 scintillating crystals [1]. The barrel portion of the ECAL $(|\eta| \mid<1.479)$ is made up of 61200 crystals, with the scintillation light read out by two avalanche photodiodes (APD) in parallel, mounted on the rear face of the crystals. The signals from groups of 5 crystals are processed by a very front-end card (VFE) which performs the preamplification and analog to digital conversion. Five VFEs are connected to each front-end (FE) card.

Figure 1

The Existing ECAL Front End System

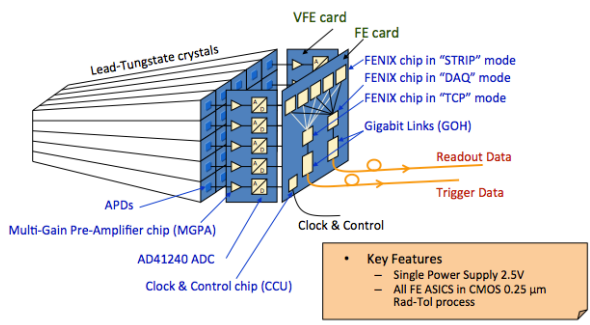

(a) Current ECAL Barrel readout electronics design. Signal shaping is done by the very front-end (VFE) card. Currently trigger primitives are generated by the front-end (FE) card. Separate readout for trigger and data. Maximum trigger rate out from $\mathrm{FE}$ is 150 $\mathrm{kHz}$ set by the current level 1 trigger accept.

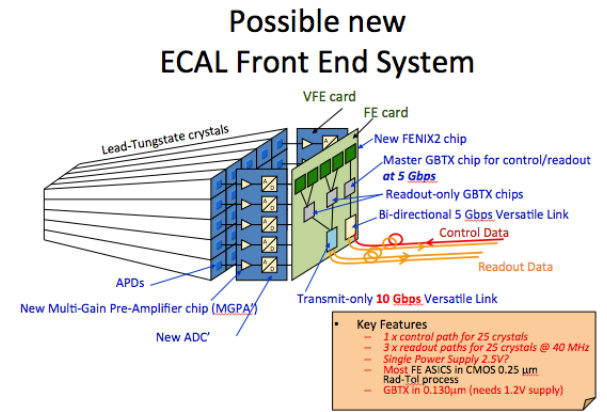

(b) Proposal for upgraded ECAL Barrel readout electronics design. Signal shaping is done by the very front-end (VFE) card. Front-end (FE) card sends single crystal information off-detector at $40 \mathrm{MHz}$. Trigger rate can be increased to $1 \mathrm{MHz}$ required by upgraded level 1 trigger accept.

Trigger primitives are calculated by the FE by summing the energy in a $5 \times 5$ array of crystals and this sum is sent to the central CMS trigger system at $40 \mathrm{MHz}$. Data are read out at the single crystal (61200 channels) level at a maximum rate of $150 \mathrm{kHz}$. There are two optical links on the FE card-one for data readout and one for trigger readout.

\subsection{Motivation for ECAL barrel electronics upgrade}

The components of the ECAL barrel can survive the HL-LHC radiation environment, but the electronics cannot meet the triggering requirements of the CMS detector for this run period (see 
section 2). Replacement of the electronics allows opportunities to improve the performance of the ECAL barrel in the high pileup and high luminosity environment of the HL-LHC.

\section{ECAL barrel trigger for HL-LHC}

The CMS trigger will have a level 1 accept rate of $1 \mathrm{MHz}$ and require a latency of up to $20 \mu \mathrm{s}$. The current FE card cannot satisfy these requirements. By replacing the FE, the optical links, and the off-detector electronics the ECAL will use the same data stream for trigger and data, sending all 61200 channels off detector at $40 \mathrm{MHz}$. This allows the trigger primitives to be calculated offdetector. The new electronics will benefit from development of the Versatile Link and GBTX at CERN. Figure $1 \mathrm{~b}$ shows a possible upgrade of the electronics.

\section{Improved online anomalous event mitigation}

Anomalous events (spikes) are energy deposits directly into the bulk of the APD (instead of interacting and scintillating in the crystal first). The spike rate is proportional to instantaneous luminosity. The spike pulse shape is shorter in time than the normal scintillation signal and also produces a signal in only one APD whereas an electromagnetic shower typically deposits at least $15 \%$ of its energy in neighboring crystals [1].

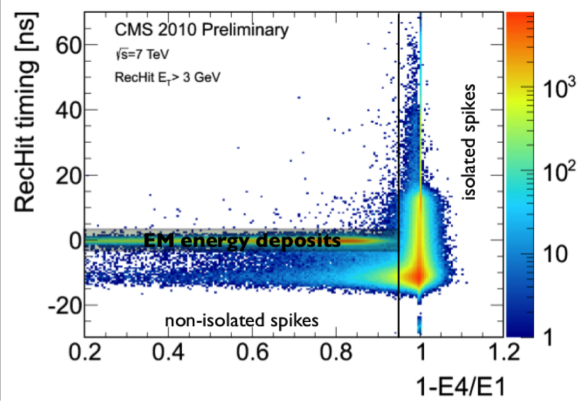

Figure 2: The two main techniques currently used for spike filtering: timing cuts measured from pulse shape and shower shape variable comparing energy in most energetic crystal to its four neighbors.

Being able to improve the spike filtering at the trigger level will reduce the fake trigger rate for ECAL at the HL-LHC considerably. Upgrading the VFE will allow timing (currently used offline) to be improved and used at the trigger level to veto spikes. The full event readout at $40 \mathrm{MHz}$ will allow the shower shape variable (currently used offline) to be used at the trigger level. This allows the ECAL to minimize the effect of spikes at the trigger level using techniques developed offline in run 1.

\section{Pileup mitigation}

In the high-pileup environment of the HL-LHC the current pulse shaping in ECAL will be distorted by pileup events from previous bunch crossings. The pulse shape produced by the VFE 
in ECAL is $42 \mathrm{~ns}$, while the bunch spacing is $25 \mathrm{~ns}$ at the HL-LHC. Energy deposited in a crystal from earlier bunch crossings could contaminate the signal. Shortening the signal shaping time by upgrading the VFE can help to mitigate this out-of-time pileup effect.

\section{APD noise and cooling}

The APDs have intrinsic noise due to the dark current in the silicon bulk from irradiation damage proportional to the square root of integrated luminosity. Extrapolations from run 1 show the noise level at the end of the HL-LHC is $400 \mathrm{MeV}$ per crystal. After $1000 \mathrm{fb}^{-1}$ this noise dominates the energy resolution. It is possible to decrease the dark current by a factor of 2 by cooling the ECAL from $18^{\circ} \mathrm{C}$ to $8^{\circ} \mathrm{C}$. By upgrading the VFE and shortening the signal shaping time the noise will also decrease.

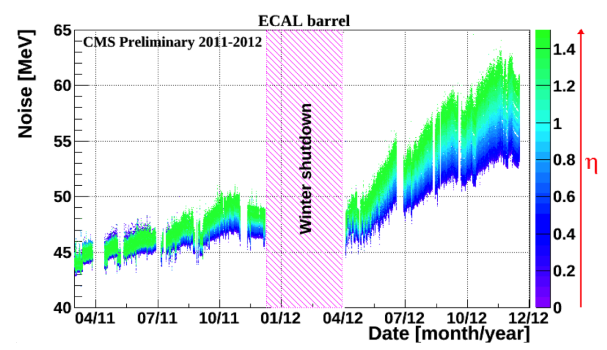

Figure 3: Noise in ECAL barrel during run 1. Noise scales as the square root of integrated luminosity. Conversion from $\mathrm{ADC}$ counts to $\mathrm{MeV}$ includes degradation of light yield due to crystal transparency loss.

\section{Conclusion}

The HL-LHC will necessitate changes to the ECAL barrel electronics. The FE must be upgraded to allow single crystal readout at $40 \mathrm{MHz}$ (full ECAL granularity for triggering). All buffers and triggering are moved off detector, which will allow future proofing. Applying these upgrades also provides an opportunity to improve performance in the high luminosity and high pileup environment. Upgrading the VFE will allow shorter signal shaping time to mitigate pileup and noise. The anomalous signal filter used at trigger level can also be improved. The planned upgrades to the CMS ECAL Barrel will allow the CMS ECAL to continue functioning well during the extreme conditions of the HL-LHC.

\section{References}

[1] The CMS Collaboration,"The CMS experiment at the CERN LHC". JINST,3 (2008). S08004, doi:10.1088/1748-0221/3/08/S08004.

[2] F. Nessi Tedaldi, “The CMS experiment at the CERN LHC”. 2012 IEEE Nuclear Science Symposium Conference also available as CMS-CR-2012/296 [arxiv.org/pdf/1211.3885v1.pdf].

[3] W Bialas and D A Petyt,"Mitigation of anomalous APD signals in the CMS ECAL". JINST,3 (2013) C03020, doi:10.1088/1748-0221/8/03/C03020 